Corporación Universitaria del Caribe - CECAR ISSN: 2422 - 085X

Enero - Diciembre 2015

Sincelejo, Colombia
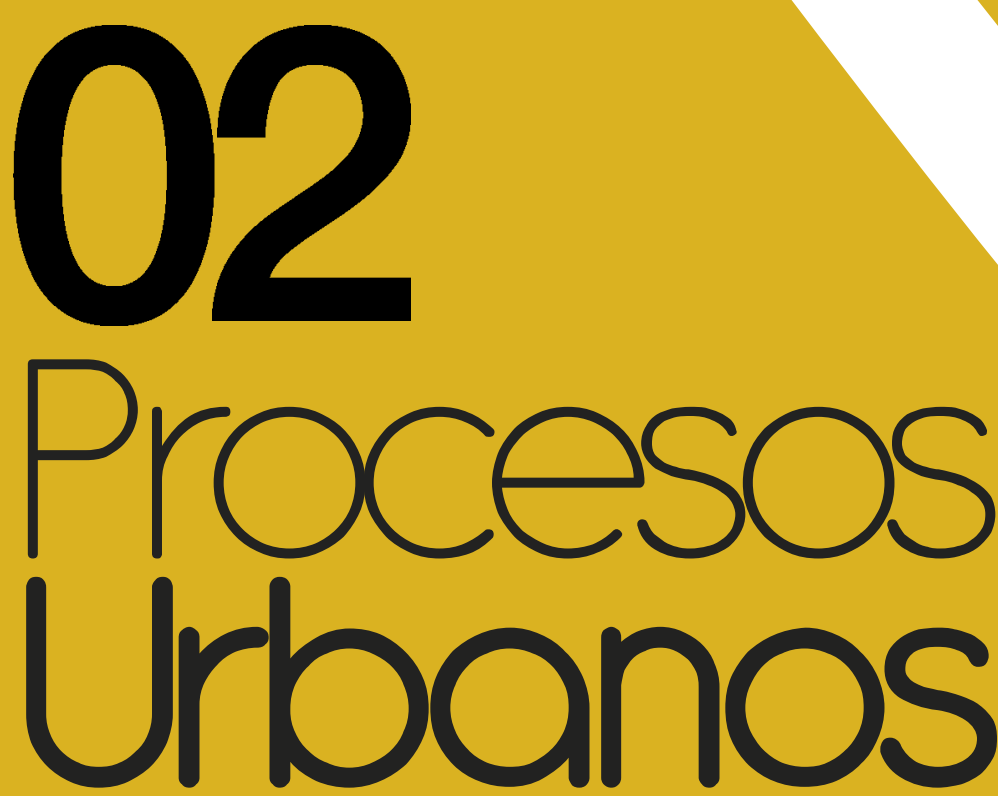

Revista de divulgación científica

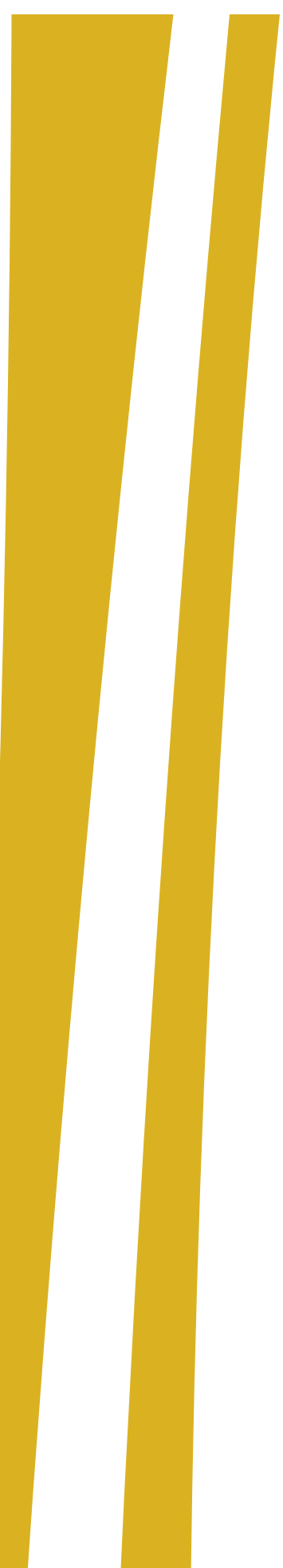

Facultad de Ciencias Básicas, Ingeniería y Arquitectura

Grupo de investigación: Teoría e historia de la arquitectua y la ciudad. 

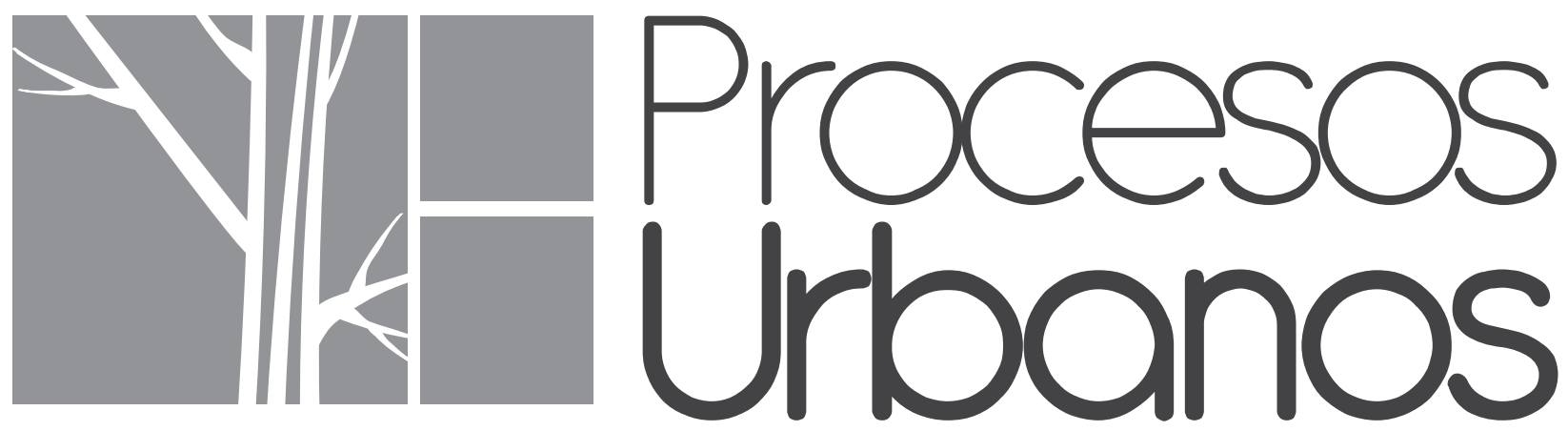

Revista de divulgación científica

Facultad de Ciencias Básicas, Ingeniería y Arquitectura. Grupo de investigación: Teoría e historia de la arquitectura y la ciudad 
Revista Procesos Urbanos

Facultad de Ciencias Básicas, Ingeniería y Arquitectura.

ISSN: 2422-085X (papel)

ISSN: $2500-5200$ (on line)

Sincelejo, enero - diciembre de 2015

\section{Piedad Martínez Carazo}

Rectora

\section{Lidia Flórez de Albis}

Vicerrectora académica

Jhon Víctor Vidal

Vicerrector de Ciencia, Tecnología e Innovación

\section{Pedro Arturo Martínez Osorio}

Editor

\section{Gilberto Martínez Osorio \\ Francisco Martínez Ochoa \\ Coeditores}

\section{Comité Editorial:}

PhD. Alexander Niño Soto. Universidad del Norte

PhD. Jorge Ramírez Nieto. Universidad Nacional de Colombia

Msc. Juan Carlos Pergolis. Universidad Católica de Colombia

PhD. Piedad Martínez Carazo. Corporación Universitaria del Caribe, CECAR

PhD. Emiro F. Martínez. Universidad de York, Canadá

\section{Comité Científico:}

Msc. Gloria Aponte García. UPB, Medellín

PhD. Jorge Gómez Ricardo, Universidad del Magdalena

Msc. Gilberto Martínez Osorio, Corporación Universitaria del Caribe, CECAR

PhD. Alfredo Otero Ortega, Corporación Universitaria del Caribe, CECAR

\section{Editorial CECAR}

Libia Narváez Barbosa

Coordinadora editorial

\section{Eduardo Támara Galván \\ Corrector de estilo}

\section{GRÁFICAS DEL CARIBE S.A.S.}

Diseño gráfico

Cra. 1B No. 40-42 Montería Tel. (57) (4) 7826622 Telefax (57) (4) 7817112

Email: diseno@graficaribe.co

Dirección:

Carretera Troncal de occidente

Kilómetro 1 vía a Corozal

Tel: 280402928040172804018 Ext 1115

www.cecar.edu.co

http://revistas.cecar.edu.co/procesos-urbanos

http://ojs.cecar.edu.co/procesos-urbanos

PROCESOS URBANOS hace parte de:

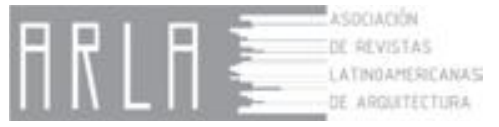




\title{
IMPACTO SOCIOECONÓMICO Y AMBIENTAL: ISLA LA LOMA MARGEN OCCIDENTAL DEL RIO MAGDALENA EN BARRANQUILLA
}

\author{
Socio-economic and environmental impact: isla la loma at rio Magdalena west margin \\ in Barranquilla
}

Alfredo Otero Ortega ${ }^{1}$

Recibido: 30 de septiembre de 2015. Aceptado 30 de octubre de 2015

\section{RESUMEN}

Con una idea de diseño inspirada en la conexión entre el rio y la ciudad, el gobierno local del distrito de Barranquilla propone desarrollar un megaproyecto urbano en una de las islas que componen el estuario deltaico de la desembocadura del rio grande de la Magdalena. Esta iniciativa, si bien está definida como una oportunidad, también es cierto que propiciará impactos de orden cultural en los habitantes, puesto que el suelo utilizado para el proyecto ha permanecido como soporte natural y defensa contra inundaciones al servir como reservorio de agua y barrera de protección a la ciudad ya edificada. Este artículo presenta los resultados parciales obtenidos en el trabajo sobre la revisión de ¿Cuál es el impacto económico, social y ambiental del desarrollo urbano de la isla La Loma?, unidad ecológica localizada al norte de estuario deltaico conformado por el sistema de canales hídricos del rio Magdalena. Muestra en sus avances una serie de situaciones que deben ser revisadas profundamente, permite comprender los beneficios ecológicos que resuelve devolverle a la isla su condición de reserva natural o caso contrario, transformarla en un desarrollo urbano edificado que termine generando económica y socialmente altos costos al Estado y en particular, al gobierno local del distrito de Barranquilla.

Palabras clave: Barranquilla, Espacio Público, La Loma, Gestión Urbana, urbanismo, cambio climático.

\begin{abstract}
With a design idea inspired by the connection between the river and the city, the district government of Barranquilla proposes to develop an urban megaproject on one of the islands that make up the delta estuary mouth of the Rio Grande de la Magdalena. This initiative, although defined as an opportunity, it is also true that shall foster impacts of cultural order among the inhabitants, since the land used for the project has remained as a natural support
\end{abstract}

\footnotetext{
${ }^{1}$ Arquitecto, urbanista, Universidad del Atlántico. Ph.D. en Arquitectura y MSc. en Geografía, Universidad del Zulia. especialista en planeación urbana y regional, Universidad Autónoma del Caribe. Planeación del ordenamiento territorial, Universidad del Norte. Planeación para la gestión ambiental; diseño y gestión curricular, Universidad del Atlántico. Financiamiento de ciudades con suelo urbano; cambio climático y políticas de Suelo, LINCOLN INSTITUTE. Investigador - asesor Centro de Estudios Geográficos CONDES - CDCHT, Universidad del Zulia - Maracaibo. Becario del programa UNESCO - MOST, Escuela Regional de Verano. CLAHE, Montevideo Uruguay. Catedrático en universidades del Caribe colombiano. Consultor para el desarrollo territorial y política de suelo urbano, medio ambiente y asuntos del patrimonio cultural. Autor de artículos y conferencista en eventos nacionales e internacionales. Email: alfredo. otero@cecar.edu.co
} 
and defense against floods and has served as a water reservoir and barrier protection of the built city. This paper presents the partial results of the work on the revision of "What is the economic, social and environmental impact of urban development on the island La Loma?", which is an ecological unit located north of deltaic estuary formed by the system of water channels of the Magdalena River. Also shown in its progress a number of situations that should be checked deeply, it could be understood the ecological benefits that solves the island back to its natural condition or reservation. Otherwise, to turn it into a complete urban development generating high economic and social costs to the national state and especially to the local government district of Barranquilla.

Keywords: Barranquilla, public space, La Loma, urban management, urban planning, climate change.

\section{INTRODUCCIÓN}

Los resultados obtenidos en el trabajo sobre la revisión de ¿Cuál es el impacto económico, social y ambiental del desarrollo urbano de la isla La Loma?; unidad ecológica localizada al norte de estuario deltaico conformado por el sistema de canales hídricos del rio Magdalena, muestra en sus avances parciales una serie de situaciones que deben ser revisadas profundamente, con la trasparencia necesaria, lo que permitirá comprender, entre los beneficios ecológicos que resuelve, devolverle a la isla su condición de reserva natural o transformarla en un desarrollo urbano edificado que termine generando económica y socialmente altos costos al Estado y en particular, al gobierno local del distrito de Barranquilla.

La intervención en el territorio amerita establecer un diálogo entre las bases teóricas y algunos elementos detectados en la relación "ciudad-ciudadano" (Otero, 2013), puesto que la participación activa de los agentes representantes del Estado, ejecutores de procesos constructivos de ciudad dentro del mismo territorio que estos habitan, pueden determinar la pertinencias del urbanismo en la construcción del espacio público, desde la perspectiva de la participación social, como podría ser el caso de la isla La Loma, al ofrecerla como un sitio de encuentro y disfrute para el buen vivir, sin que pierda su condición ecológica natural.

Para comprender el problema es necesario entender el papel que juega ecológicamente esta parte del estuario deltaico de la llanura de inundación del rio Magdalena próxima a su desembocadura, al servir como barrera o "colchón hídrico" de protección natural a una ciudad edificada sobre suelos muy próximos al nivel del mar. Debemos reconocer los altos costos que ha representado el desarrollo urbano a que fue sometida la isla del sector de Barranquillita, y el grave impacto ambiental y de sustentabilidad urbana que ha ocasionado a los intereses de la ciudad. Barranquilla no se inició de espalda al rio, sus primeras ocupaciones urbanas se dieron de frente al sistema de canales hídricos, que fueron el motor para el desarrollo $y$ emprendimiento económico, financiero y empresarial de los inicios de la ciudad.

Este transcurrir de la primera década del siglo XXI ha colocado frente a nuevos retos del devenir humano y hace más pertinente el diálogo de saberes que faciliten la búsqueda de acuerdos acertados, permitiéndo consolidar un planeta más amable y razonable, con relación a las diferencias existentes hasta finales del siglo pasado. Urge la necesidad de resolver el problema universal, que según Morín (1993), es cómo adquirir la posibilidad de articular y organizar las informaciones sobre el mundo, para lo cual es necesario un pensamiento ecologizante ${ }^{2}$, que sea capaz de superar esta era planetaria que se inicia y se desarrolla, en y por la violencia. 
PROCESOS URBANOS - Revista de divulgación científica vol. 2 enero - diciembre 2015 (90 - 100)

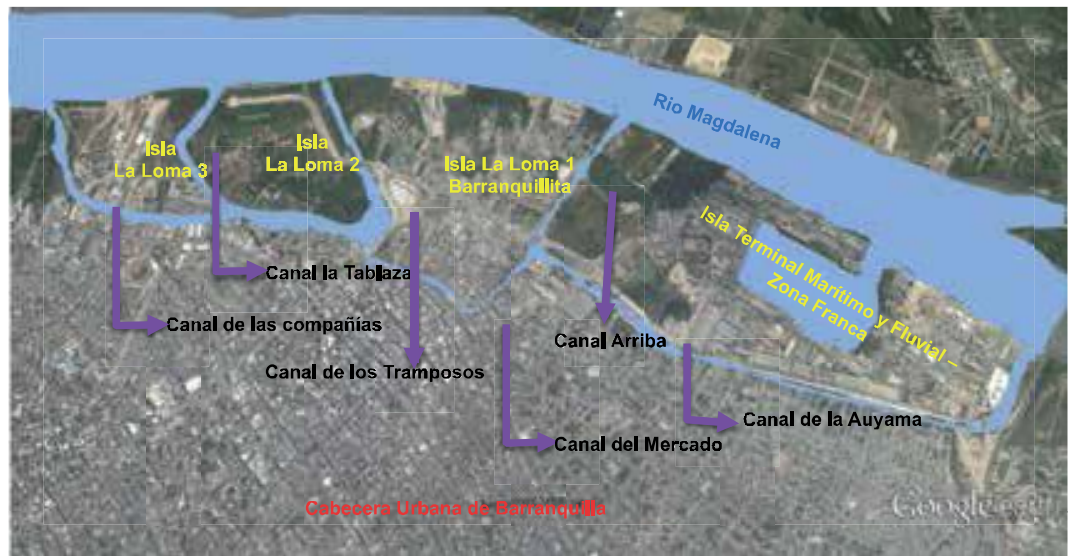

Figura 1. El Sistema de canales hídricos de Barranquilla Fuente: Elaboración propia con imágen tomada de Google earth (2014)

Propiciar obras para mejorar el hábitat, debe concebirse bajo la dinámica urbana espacial del territorio que vincule a todos los ciudadanos afectados por dichos proyectos a partir de la formulación, gestión y ejecución de los mismos, lo cual tiene que ser entendido como una manera más de democratizar los procesos de urbanización, de tal manera que facilite la interacción del urbanismo y la acción social, como herramienta pedagógica y constructora de nuevos conceptos y conocimientos, que aun complejo, permite una nueva manera de reaprender los espacios urbanos habitados, siendo una traducción y reconstrucción a partir de señales, signos, texturas, símbolos, escalas y colores, entrelazados con la participación ciudadana, para generar un nuevo pensamiento de ciudadanos comprometidos con la visión de su ciudad.
El estuario deltaico y un sistema de canales e islas del río Magdalena.

La cabecera urbana de Barranquilla se encuentra ubicada en la margen occidental del sistema de canales hídricos que hacen parte del gran estuario deltaico del río Magdalena, localizados próximos a su desembocadura en el denominado punto geográfico Bocas de Cenizas. La importancia socioeconómica del lugar radica en su capacidad hídrica mostrada desde la época de la Colonia, cuando permitió desarrollar en sus inmediaciones un asentamiento urbano que facilitaba la trashumancia en la región. A principios del siglo XX, la ciudad logró un significativo crecimiento poblacional y un desarrollo urbanístico producto del establecimiento como puerto fluvial de intercambio entre el interior del país y el resto de ciudades del planeta,

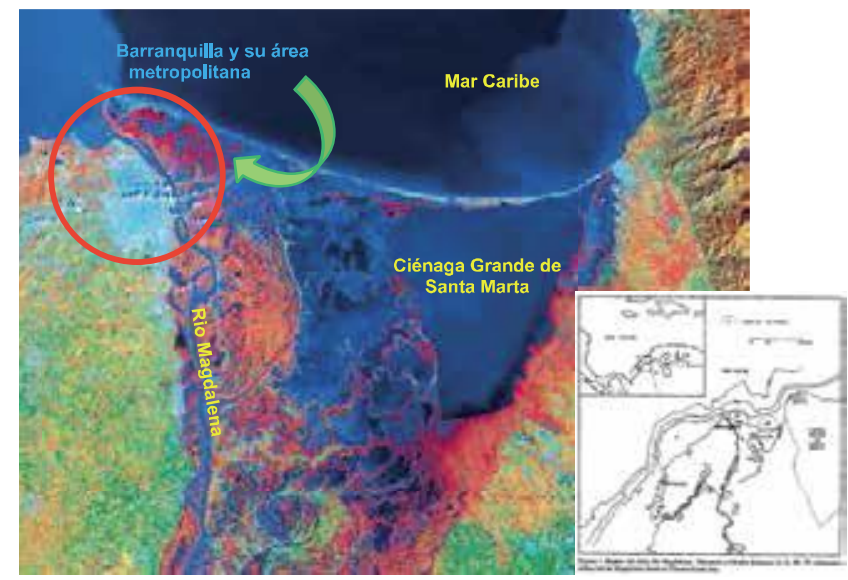

Figura 2. El Estuario deltaico del río grande de la Magdalena Imágen tomada de (Remas, 1996) Delta y estuarios de Colombia. Adaptación propia. 
que la convirtieron en un centro urbano con funciones comerciales, financieras, administrativas y culturales, entre otros de gran interés.

El estuario deltaico por lo tanto ha permitido desarrollar un modelado morfológico a lo largo de todo el sistema de canales hídricos, que históricamente ha servido para equilibrar la capacidad del caudal que tiene el río Magdalena, lo que facilita un esquema de protección propio para tierra dentro, es decir, las extensiones de territorio que se encuentran más allá de esta parte del estuario deltaico de la desembocadura del rio, que hoy día conforman la gran conurbación urbana de Barranquilla y su área metropolitana.

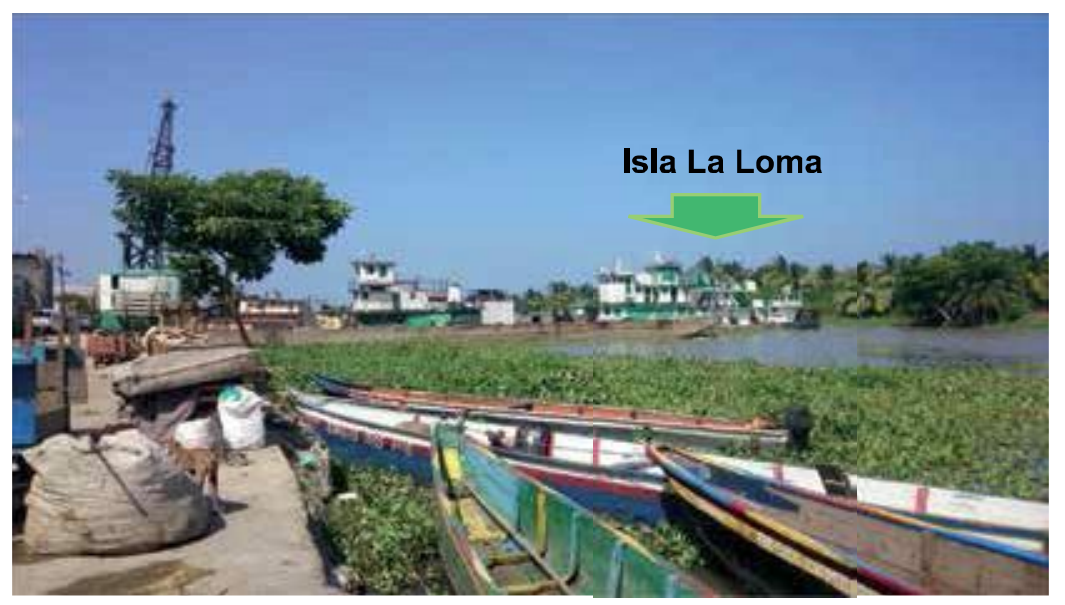

Figura 3. Localización de la unidad ecológica de la Loma Fuente: Otero 2014

La ciudad se enfrenta durante el año a caudales promedios entre los 4.000 a $10.000 \mathrm{~m} 3$ por segundo de presión hídrica del cauce del rio antes de depositar sus aguas al mar Caribe, y con ello, una carga de sedimento superior a 140 toneladas anuales que ejercen una presión sobre el sistema de canales y sus islas, amortiguando el impacto y protegiendo el territorio urbanizado sobre la planicie deltaica muy próxima a su desembocadura y con alturas inferiores a $200 \mathrm{msnm}$. El estuario ha sido propicio para la actividad comercial y productiva, y como nicho ecológico se constituye en un sitio de producción y procreación de especies que contribuyen con la estabilización ambiental del estuario. De esta manera se pueden considerar estos escenarios geográficos como un espacio para el desarrollo urbanístico, por su localización próxima al mar y el contacto con otras comunidades, siempre y cuando la utilización de las ventajas comparativas del lugar no obstruya los sistemas ecológicos.

El sistema de canales y sus islas, frente a los cuales ha crecido la ciudad se comportan como un "colchón hídrico" que amortigua las impetuosas crecientes del río. Está conformado por una serie de cauces menores, denominados caños que se ramifican sobre la planicie de esta parte del delta de la llanura de inundación y se desprenden del rio a través de diferentes bocas, que hacen ver el interés de esta geomorfología del delta, pues su capacidad de absorción y desorción de los caudales facilita la protección del sistema construido que conforma la ciudad en esta parte del territorio urbano.

El sistema de canales del río Magdalena tiene una longitud aproximada de 10 kilómetros y hace parte del sistema hídrico de la ciudad, localizado en la planicie aluvial deltaica del río Magdalena sirve de receptora de las carga de aguas lluvias que se precipitan sobre Barranquilla y su área metropolitana. Se encuentra formado por los canales de la Ahuyama $(3,7 \mathrm{~km})$, el canal Arriba $(1,5 \mathrm{~km})$, el canal del Mercado $(0,9$ $\mathrm{km})$, el canal los Tramposos $(1,2)$ el canal de la Tablaza $(1,3 \mathrm{~km})$ y Las Compañías $(2,7 \mathrm{~km})$, caño Agromar $(0,8 \mathrm{~km})$ y caño $\mathrm{C}(1,0 \mathrm{~km})$. Este sistema bordea el primer 


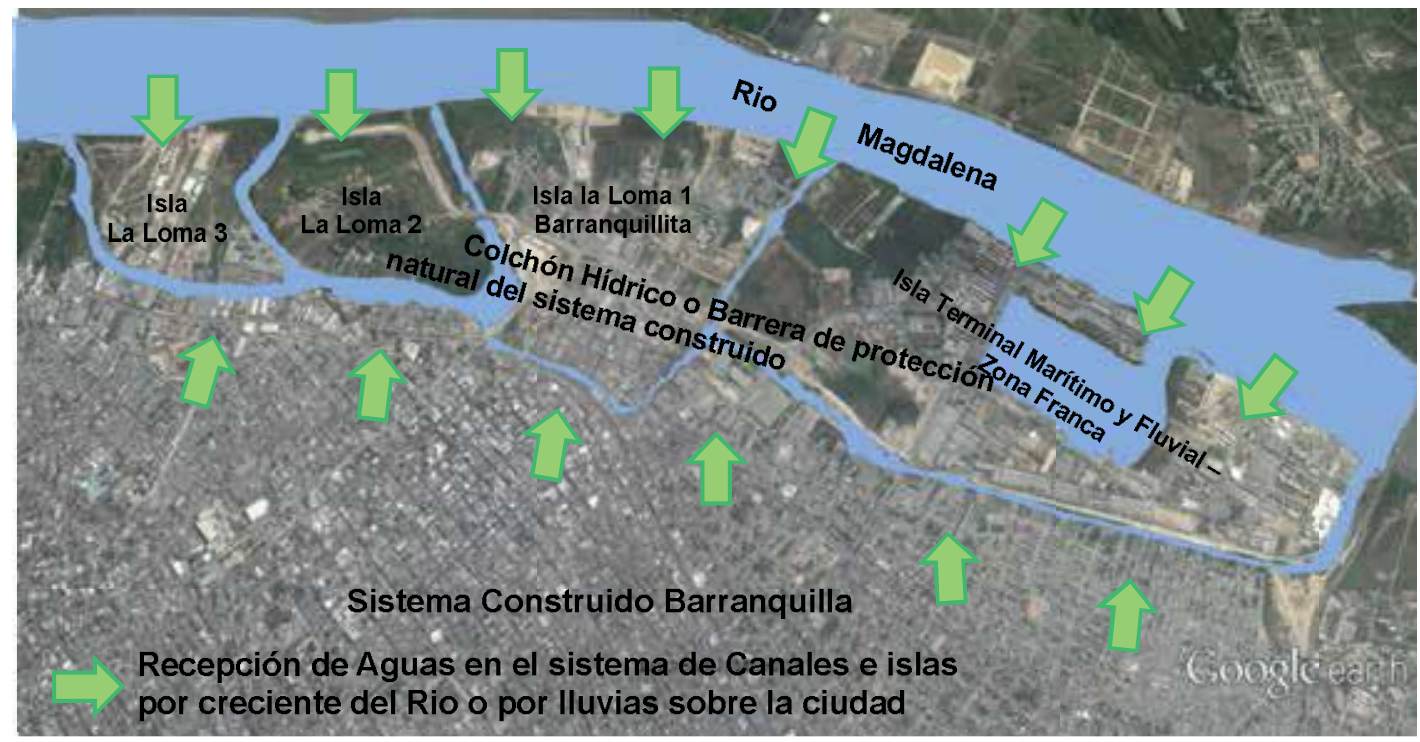

Figura 4. Colchón Hídricos de Protección al Sistema Construido de Barranquilla Fuente: elaboración propia con imagen tomada de Google earth 2014.

desarrollo urbanístico hecho en la ciudad a principios del siglo XX denominado "sector de Barranquillita", que debe en parte su deterioro ambiental, económico y físico, a los embates de las crecientes del rio por encontrarse construido sobre una de las islas del sistema, por lo que se constituye en uno de los grandes problemas sociales del centro de la ciudad.

\section{El desarrollo urbano del sector La Loma: ¿acierto o desacierto?}

Barranquilla no ha escapado de los impactos producidos por las migraciones poblacionales de la región, que atraídas por el desarrollo portuario, el comercio y la industrialización urgida de mano de obra barata, han recibido abundante personal procedente de las zonas rurales y el interior de la nación, lo cual originó formas urbanas y densidades desequilibradas por asentamientos informales $y$ barrios con insuficiencia de infraestructura de servicios públicos. Este nuevo patrón de urbanización instaurado en toda América Latina desarrolló variados componentes en la nueva estructura urbana de las ciudades, y uno de ellos fue el traslado de un modo de vida rural al ámbito urbano (Otero, 2013).

Los sectores que conforman las islas del sistema de canales hídricos del río Magdalena se han visto sometidos a este tratamiento, quizás por la falta de una política pública urbana que, desde los primeros procesos de urbanización de la ciudad, hubiesen impedido la invasión y deterioro de estas zonas de protección ambiental naturales propias del estuario deltaico. De las islas que hacen parte del sistema de caños, la que mayormente ha sido sometida a proceso de urbanización es el denominado sector de Barranquillita, la cual padece un deterioro ambiental, socioeconómico y físico. Para la CEPAL (2011), la urbanización involucra un grado significativo de artificialización del medio; su más alta densidad demográfica genera mayores presiones por unidad de territorio $y$, típicamente, por los estilos de vida y mayores ingresos de la población urbana se asocia a patrones de producción, consumo y generación de residuos más gravosos para el ecosistema.

El desarrollo urbano previsto para el denominado sector La Loma, se proyectó como una estrategia de oferta de servicios urbanos regionales, que tiene como gancho para la intervención urbanística una vía que irrumpe el sistema natural de las islas y toda la zona de protección natural del estuario deltaico que protege a la ciudad, por lo cual, el objetivo general del plan parcial que propone este desarrollo descontextualiza 
la situación de la isla, la cual no está urbanizada, al proponer adoptar normas urbanísticas que se encaminen a mejorar las condiciones ambientales del lugar.

Si bien es cierto que el poder administrativo de la nación le concesionó el estuario deltaico que protege la ciudad a los empresarios e industriales que llegaron el siglo pasado a hacer de Barranquilla la Puerta de Oro de Colombia, los orígenes de la ciudad muestran que la misma tuvo una génesis de ciudad puerto, de cara a su sistema de canales hídricos que facilitaron el fondeo de embarcaciones de manera segura y conectados al río, que favoreció la movilidad de carga y pasajeros a nivel nacional e internacional, con una intendencia fluvial localizada en tierra adentro, tal cual lo demandan los embates propios de la naturaleza del río Magdalena, puesto que el "colchón hídrico" formado por las islas y los caños han sido y siguen siendo el punto de equilibrio que protege a la ciudad de las creciente propias del gran caudal del río.

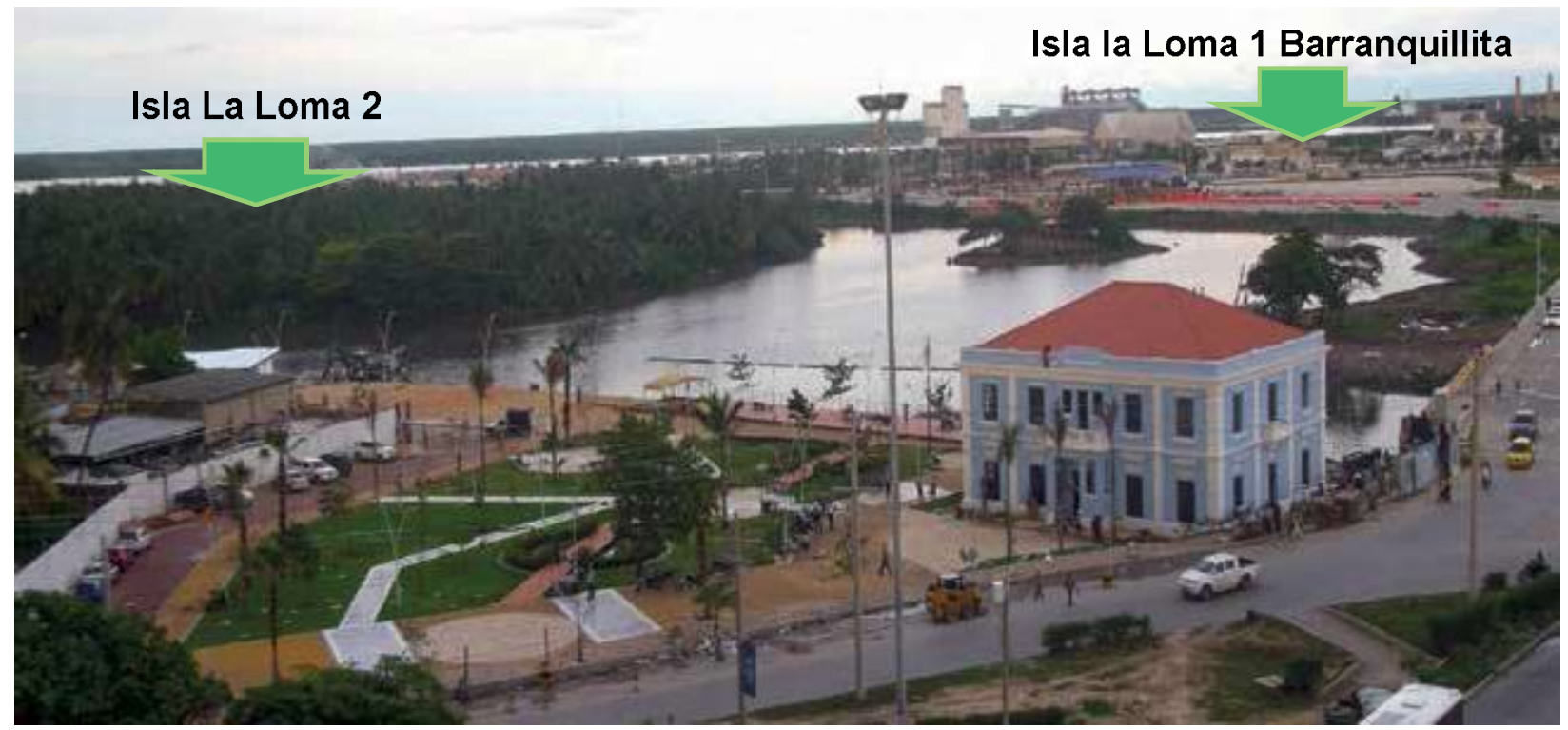

Figura 5. Renovación urbana alrededor de la isla La Loma. Intendencia fluvial. Fuente: imagen tomada de http://www.skyscrapercity.com

¿Por qué ahora se pretende someter a la isla La Loma a un desarrollo urbanístico? Hacerlo es potenciar el deterioro del ecosistema del estuario que ha estado protegiendo este asentamiento humano.

Conforme a lo adoptado en el Decreto 123 de 2005, el plan parcial para los sectores de Barranquillita - La Loma - Barlovento establece una acción urbanística de nuevo desarrollo para la isla La Loma, localizada en el norte del área que enmarca el sistema de canales del río, con un área aproximada de 110 ha, donde se determinaron dos sectores normativos denominados: Sector turístico La Loma. SN 11 y Sector residencial La Loma. SN 12. Esta decisión irrumpe con el ecosistema, al proponer alterarlo con unos índices de ocupación del suelo que determina otros índices de construcción, lo que pone en riesgo el ecosistema y las zonas naturales de protección que han servido para proteger la ciudad.

¿Será que lo ocurrido con la isla donde se desarrolló Barranquillita aún no explica el deterioro ambiental y los bajos índices de sustentabilidad urbana del sector?

\section{Impacto económico, ambiental y social} del desarrollo urbano de la isla La Loma Después de la conferencia internacional de Población y Desarrollo del Cairo en 1994 y la reunión regional de América Latina y el 


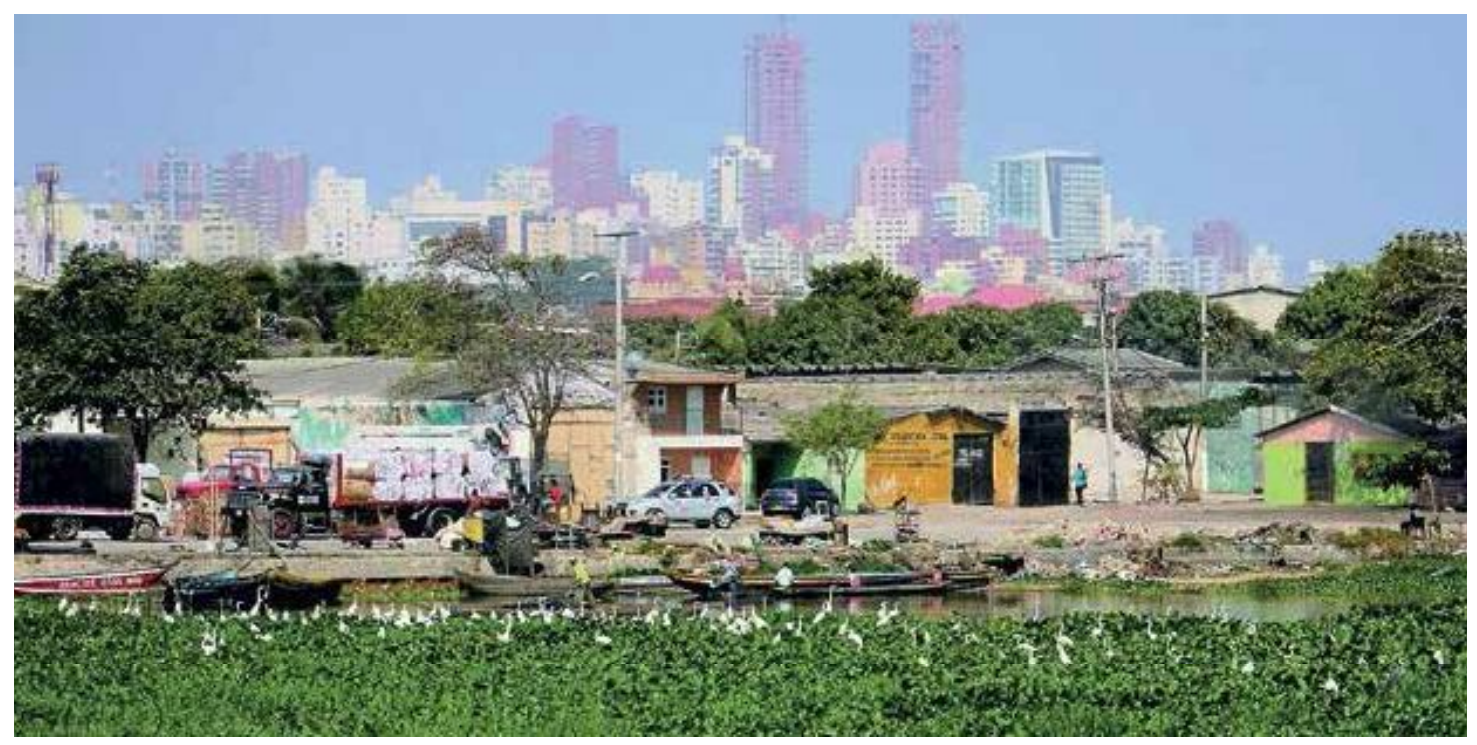

Figura 6. Vista de Barranquilla desde la isla La Loma. Fuente: Christian Mercado.

Caribe preparatoria de la conferencia de las Naciones Unidas sobre los asentamientos humanos (Hábitat II) realizada en Santiago de Chile en 1995, en Colombia se ha venido diseñado nuevas políticas de planeación que permiten entender que esta no asume validez sin tener como premisa importante a la población, razón por la cual se adopta, como principio fundamental del desarrollo humano sostenible, que la población es el centro de las preocupaciones y constituye el sujeto y el objeto de las acciones del desarrollo, para así una visibilidad a la relación de lo social y lo urbano como elementos integradores a las acciones e intervención del territorio habitado por parte del Estado político desde sus gobiernos, llámense nacional, regional o local.

Frente a la posibilidad de llevar a cabo los procesos de urbanización contemplados en el marco normativo por los Decretos 154 de 2000, 123 de 2005, 131 de 2007, 198 de 2014, y el Acuerdo 003 de 2007, que intervienen, entre otros, la isla La Loma, se han revisado las posibles incidencias de tipo social, económico y ambiental que impactarán en un mediano y largo plazo al sector mencionado, lo que pone en riesgo la ya contaminada zona de reserva natural, al ver afectado el nicho biológico que proporciona un ambiente apropiado para el desarrollo de especies ecológicas propias de un estuario deltaico, como el de la llanura de inundación, la cual está formada por lodos limosos y arenas finas grises oscuras enriquecidas con materia orgánica (Martínez y Molina, 1992; citado en Bernal 1996), presente en el sistema de canales hídricos de Barranquilla constituido por caños, diques y con presencia de pantanos lagunares.

El dilema del urbanismo actual, según Borja (2003), acompaña a los procesos desurbanizadores 0 disolutorios de la ciudad mediante respuestas puntuales, monofuncionales o especializadas, que se expresan por medio de políticas sectoriales sometidas al mercado y ejecutadas por la iniciativa privada. O si, por el contrario, impulsa políticas de ordenación urbana y de definición de grandes proyectos que contrarresten las dinámicas perversas y que se planteen el hacer ciudad favoreciendo la densidad de las relaciones sociales en el territorio, la heterogeneidad funcional de cada zona urbana, la multiplicación de centralidades polivalentes y los tiempos y lugares de integración cultural.

El impacto de un posible desarrollo urbano de la isla La Loma se verá reflejado desde dos escenarios futuros (i: la sostenibilidad y ii: la sustentabilidad del ecosistema urbano), en las cuales el balance o equilibrio 
entre las metas económicas, sociales y ambientales deben ser asumidas más allá de la definición acuñada por el informe Brundtland $^{3}$. Para Gudynas (2010), una lectura atenta de esa definición demuestra que hay varios componentes en juego: (i) se encuentra el compromiso con las "generaciones futuras", pero, (ii) enseguida se abordan los "límites al desarrollo". Si bien se los acepta, se sostiene que son relativos, y en consecuencia se puede manejar tanto la tecnología como la organización social, frente a los determinantes ecológicos.

La definición del informe Brundtland debe ser entendida en una secuencia histórica, inserta en los debates sobre los límites ecológicos al crecimiento. Frente a ese populismo simplista surge la segunda estrategia mundial de la conservación EMC-II", conocida con el lema "Cuidar la Tierra". En ella se admite que la definición de sustentabilidad del reporte Brundtland generaba confusiones debido a que se "han utilizado indistintamente 'desarrollo sostenible', 'crecimiento sostenible' y 'utilización sostenible', como si sus significados fueran idénticos, y no lo son". Enseguida se advierte que "crecimiento sostenible" es un término contradictorio: nada físico puede crecer indefinidamente. La definición de desarrollo sostenible que ofrece la EMC-II es más breve, pero tiene un sentido más preciso: "mejorar la calidad de la vida humana sin rebasar la capacidad de carga de los ecosistemas que la sustentan".

A partir de esta conceptualización de la sustentabilidad del ecosistema, este estudio considera que un proyecto de nuevo desarrollo urbano en una zona de reserva natural, si bien puede generar nuevos escenarios para el encuentro y la convivencia social de la ciudadanía, estos no pueden sobre pasar la capacidad de carga del suelo de protección, y debe garantizar el ciclo de vida del nicho biológico que le pertenece. La isla no está urbanizada, por lo tanto la acción social debe realizarse desde afuera, para evitar que el ecosistema del estuario deltaico siga siendo deteriorado, mas aún, sabiéndose de la construcción de aproximadamente 1.8 kilómetros de vías (avenida del río) y un mirador (Malecón) sobre un terraplén construido en la margen del río de una longitud de 500 metros lineales. El posible impacto debe ser conciliado a partir de una política pública urbana incluyente que favorezca los sistemas estructurantes del espacio público y los componentes ambientales para permitir que un proyecto urbano en la isla no vaya más allá de acercar a la ciudadanía a sus recursos naturales.

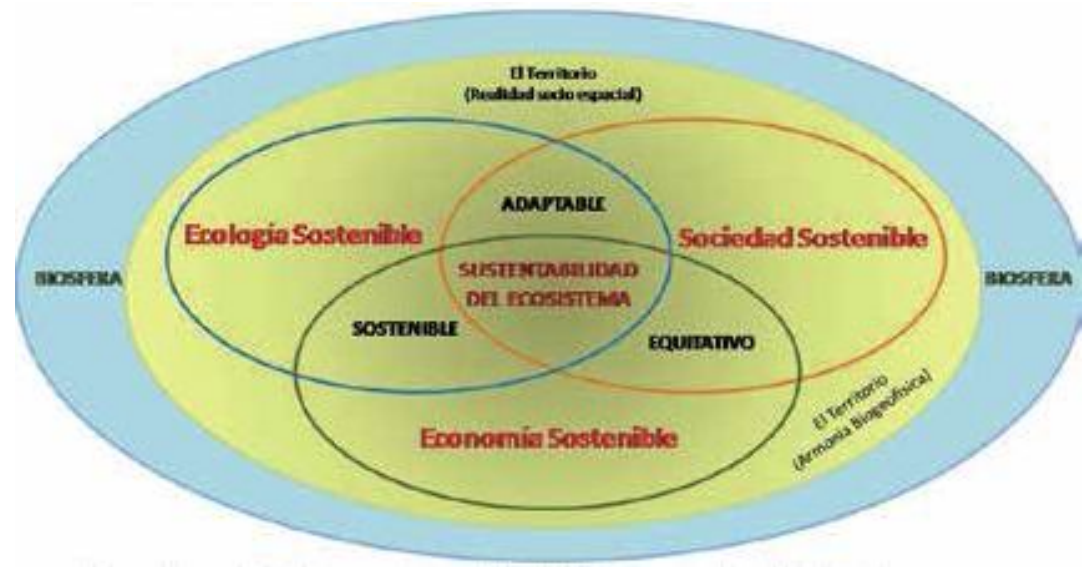

Figura 7. Desarrollo ecológico sustentable - Cuidar la Tierra.

Fuente: Estrategia Mundial de la Conservación (EMC - II 1991) Elaboración propia, 2013.

\footnotetext{
3WCED (1978). Our Common Future, World Commission on Environment and Development, Oxfort University Press, Oxford. Esta comisión estuvo dirigida por la doctora noruega Gro Harlem Brundtland, quien entre los años 1986 y 1989 se desempeñó como primera ministra.

${ }^{4}$ La segunda segunda estrategia mundial de la conservación celebrada en 1991 conjuntamente con el PNUMA y el WWF es responsable del documento "Cuidar la Tierra".
} 
PROCESOS URBANOS - Revista de divulgación científica vol. 2 enero - diciembre 2015 (90 - 100)

Lo paradójico en la intervención de esta reserva natural se constituye en desconocer los costos ambientales que le cuesta a la ciudad y sus habitantes, la desaparición de este espacio ecológico y la destrucción sistematizada del nicho biológico propio de un estuario deltaico, mientras que desde la normativa del plan ya se está haciendo un estimado equitativo de cargas y beneficios para los inversionistas y urbanizadores. Los beneficios estarán representados en los usos y el potencial de desarrollo que se da para cada uno de los predios incluidos en el sector, resultantes del reajuste de tierras e integración inmobiliaria a que haya lugar.

Generar impacto que incida potencialmente en la recuperación del bosque, incluyéndolo como una estructura de composición y diseño paisajístico debe ser un objetivo dentro de las opciones existentes de intervenir para el encuentro y la convivencia ciudadana con la naturaleza. Parte de esta estructura debe impactar en la recuperación de las rondas hídricas de todo el sistema de canales de las islas, y el desarrollo urbano implantado debe limitarse a definir áreas de conservación y protección para la sostenibilidad del aprovisionamiento de agua para el tratamiento humano y la inclusión en medio del paisaje natural de rutas, senderos, ciclo vías y parque de bajo impacto ambiental, para hacer posible alcanzar los máximos estándares de calidad en provisión de zonas verdes y la conexión al sistema de espacio público de la ciudad.

La convivencia ciudadana, como valor de impacto socioeconómico y ambiental debe ser garante de un nuevo modo de vida urbano compatible con los ecosistemas de la ciudad. El espacio público debe darse bajo la premisa de resolver un problema patológico del urbanismo de las ciudades con grandes deterioros, y el mismo debe recibir e integrarse a los cambios propios del proceso de transformación que va marcando la planeación del ordenamiento (Otero, 2013). El espacio público dado desde la participación de la ciudadanía debe fortalecer los lazos entre los actores de gobierno y las comunidades beneficiarias, para hacer posible la construcción de una cultura de interpretación de los lugares urbanos y los objetos arquitectónicos que la componen hasta convertir en hábito el vivir, convivir y buen vivir la ciudad.

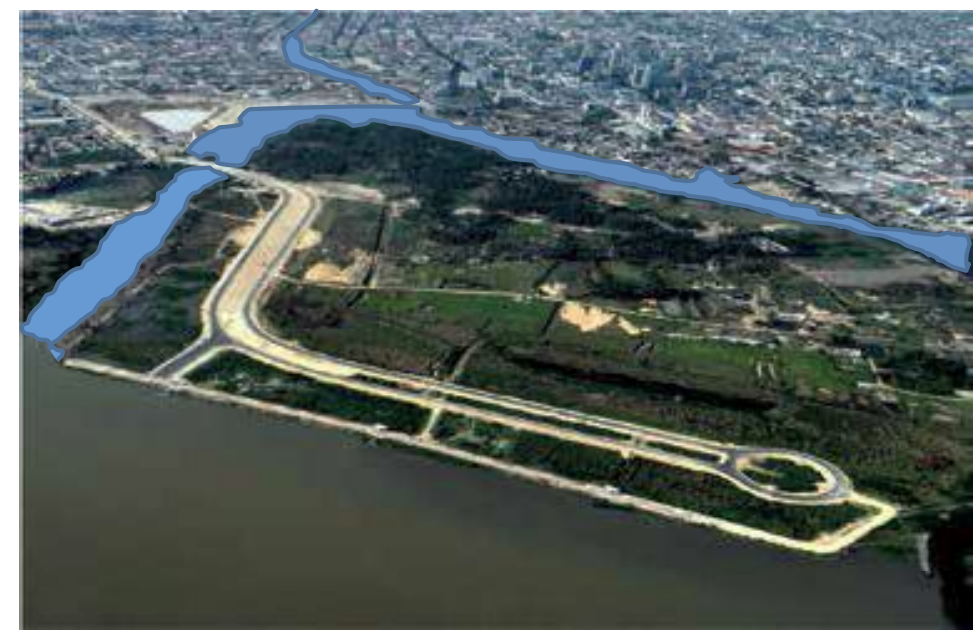

Figura 8. El desarrollo urbano del sector La Loma: ¿acierto o desacierto?. Aerofotografía: estado actual de la isla La Loma (2014)

Fuente: edubar.com.co

\section{CONCLUSIONES}

El reto debe ser producir nuevas visiones para el desarrollo de la región Caribe colombiana, desde la estructuración de un modelo de ciudad que facilite el equilibrio del territorio y favorezca la solución de condiciones de habitabilidad de las mismas, calidad en los servicios e infraestructura suficiente, equipamiento, vivienda y la dinámica de un crecimiento 
urbano equilibrado en lo económico y social, fundado en un desarrollo sustentable.

La articulación de lo social a los procesos de urbanización es una estrategia que favorece las condiciones para minimizar los impactos que produce una intervención en un territorio cualquiera. El fenómeno del urbanismo y la participación ciudadana en la intervención del desarrollo de las ciudades debe ser abordado como una parte del todo que conforma la complejidad misma de la vida cotidiana de una ciudad y sus ciudadanos y ciudadanas. Es por esto que se debe interpretar y entender las nuevas dinámicas sistémico - complejas de la sociedad, las cuales desde el pensamiento y las ciencias de la complejidad, como la biótica global y profunda, el ambientalismo holístico, y un pensamiento ecologizante puedan permitir aprehender con mayor fuerza heurística los fenómenos sociales con los cuales se convive.

Sería difícil hallar a alguien - ya sea entre académicos, activistas comunitarios y tanto más entre los hombres y mujeres de la calle, que no esté de acuerdo con la importancia social de la vida cotidiana. Y, no obstante, esa vida cotidiana, en muchos de sus aspectos y rasgos sigue siendo para la teoría social una especie de "Cenicienta" (Espina, et al., 2006), razón por la cual el Urbanismo es una disciplina llamada a servir, no solo para propiciar el desarrollo urbanístico, de diseño y edificación de los pueblos, sino también la integración de estos como cosa construida, con todo aquello que lo habita y que puede suplir sus necesidades.

El urbanismo social, como herramienta pedagógica de trasformación ciudadana se desarrolla como concepto pedagógico a partir de la transdisciplinariedad para comprender la realidad urbana de la ciudad y poder en conjunto con las comunidades iniciar la construcción de un nuevo pensamiento de ciudad con unos ciudadanos y ciudadanas que re-aprendan el conocimiento de la misma. Otero (2010), lo define como un instrumento de transformación a través del cual se materializan y viabilizan las diferentes formas de desarrollo de la ciudad y se propende por intervenciones integrales que incluyan funcionalidad y estética con un propósito dignificante $e$ incluyente $y$ renovador de una nueva visión y concepto de ciudadano y ciudad.

El desarrollo urbanístico ha demostrando un alto consumo de energía, recursos naturales y afectación de suelos por cada una de sus intervenciones, además de ser uno de los responsables de las emisiones que producen el cambio climático global, razón por la cual se hace necesario producir nuevas dinámicas en los procesos de planeación del orden urbano, como en los diseños arquitectónicos y la producción de materiales que permitan generar procesos limpios y un desarrollo urbano sostenible.

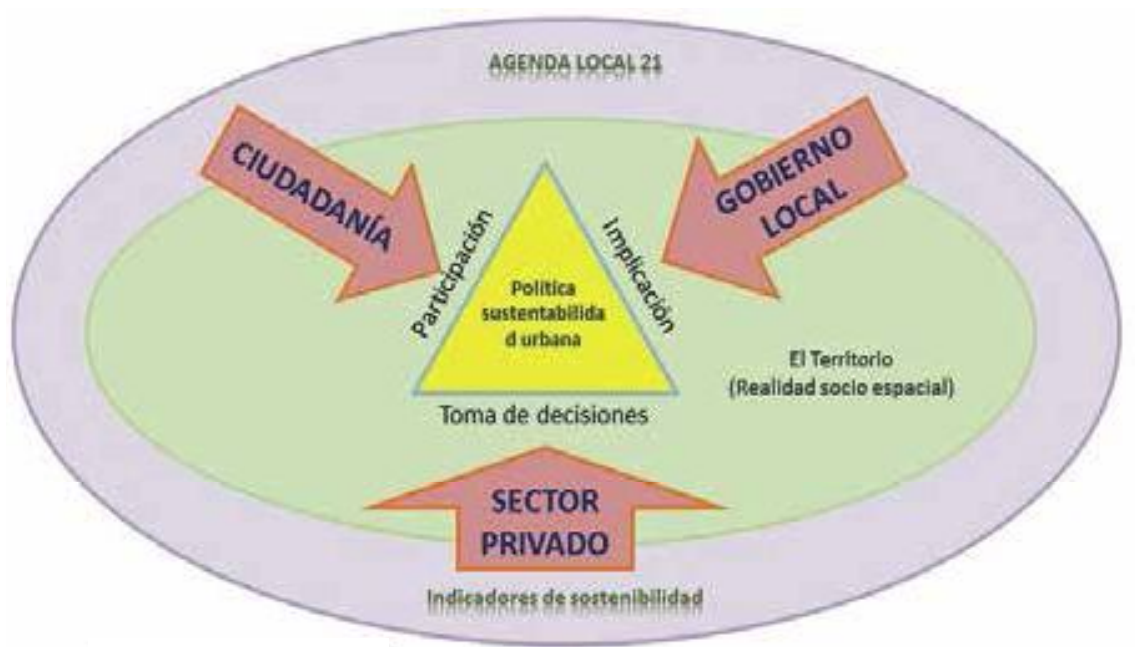

Figura 9. Políticas para la gestión de la sustentabilidad urbana Fuente: Elaboración propia, 2013. 
PROCESOS URBANOS - Revista de divulgación científica vol. 2 enero - diciembre 2015 (90 - 100)

La ciudad emerge, según Fontalvo (2008), no como lo homogéneo, reductor de las partes al todo, sino más bien deviene en un sistema complejo de mutuas interdependencias sociales, que hacen pensar a la ciudad como una forma de vida inevitable, es decir, como un problema de la condición humana que no se reduce a lo programado, sino que le da juego a una ciudad diferenciada.

Lo urbano, lo social y lo humano son tres elementos que se encuentran constantemente compartiendo el espacio habitable y su interacción e interdependencia ocurren en medio de un entorno endógeno, lo que favorece una serie de alternativas emergentes que lleva a reflexionar, estudiar y evaluar los procesos complejos de la ciudad como elemento propiciado por el urbanismo. El análisis sobre el impacto que puede producir un desarrollo urbano es de interés colectivo, pues de él depende la sustentabilidad urbana del territorio que se habita y el mismo que habitarán futuras generaciones.

\section{REFERENCIAS}

Bernal, G. (1996), Caracterización geomorfológica de la llanura deltaica del río Magdalena con énfasis en el sistema lagunar de la ciénaga grande de Santa Marta, Colombia. Documento disponible en:

http://www.scielo.org.co/scielo. php?script $=$ sci_arttext $\&$ pid $=$

S0122-97611996000100002. Consultado julio 2013.

Borja, J. (2003) "Ciudadanía y espacio público" revista Ambiente y Desarrollo. Septiembre 1998. Vol XIV No 3 pp 13 - 22. ISSN 0716 - 1476.

CEPAL, (2011), Población, territorio y desarrollo sostenible. Documento disponible en: http://www. cepal.org/celade/noticias/ paginas/0/46070/2012-96-poblacionweb.pdf. Consultado septiembre 2012.

Decreto $N^{\circ} 198$ del 21 de febrero de 2014, por el cual se delimita la unidad de actuación urbanística complejo habitacional "La Loma", sector normativo 11, sector turístico La Loma, SN-11. y, sector residencial La Loma, SN-12 del plan parcial Barranquillita La Loma - Barlovento.

Decreto $N^{\circ} 131$ del 21 de septiembre de 2007, por el cual se delimita la unidad de actuación urbanística estación central del distrito industrial y portuario de Barranquilla.

Decreto $N^{\circ} 123$ del 12 de agosto de 2005, por el cual se adopta el Plan parcial para los sectores de Barranquillita - La Loma - Barlovento del distrito industrial y portuario de Barranquilla.

Decreto $N^{\circ} 154$ del 6 de septiembre del 2000, por el cual se adopta el Estatuto urbano distrital del distrito industrial y portuario de Barranquilla.

Espina, M.; Sotolongo P. y C. Delgado (2006). La revolución contemporánea del saber y la complejidad social. CLACSO Libros, colección Campus Virtual, Buenos Aires.

Fontalvo, R. (2008). Educar en la complejidad. Barranquilla. Ed. Universidad Simón Bolívar.

Gudynas, E. (2010). La ecología política de la crisis global y los límites del capitalismo benévolo. Íconos ediciones. Quito, Ecuador.

Morín, E. (1993), Tierra patria. Barcelona. Ed. Kairos, S.A.

Odum, E. y Sarmiento, F (1998), Ecología: El puente entre la ciencia y la sociedad. Mc Graw Hill, Interamericana.

Otero, A. (2013), Espacio público y cultura ciudadana: pertinencia para un urbanismo social. Memorias del 1er seminario de investigación urbana. Edición Universidad del Zulia. ISBN 978-980-402-144-2.

Otero, A. (2010), "La pertinencia de un urbanismo social para un nuevo concepto de ciudadanía". En Ekistics, Revista de la Facultad de Arquitectura, Arte y Diseño de la Universidad Autónoma del Caribe. Vol. 1, No. 1. Enero-diciembre; pp.74-81. 